\title{
Time-window Complexity and its Application in the Fault Diagnosis of Bearing
}

\author{
Pei JIANG ${ }^{1 a}$, Liang GAO ${ }^{1}$
}

${ }^{1}$ Post doctoral mobile station of mechanical engineering, Huazhong University of Science and Technology, 430033, Wuhan Hubei, China

\begin{abstract}
Different from conventional spectral method, complexity analysis treaded with the signals' time domain structure feature. Before, due to the no stationary and the unevenness of state space of mechanical signals, the state information is likely to get a loss. Now, the time-window complexity is proposed to overcome certain limitations of complexity itself in some extent. It will help to extract the state features of mechanical systems in different states. The concept and algorithm of time-window complexity is introduced detailed. The way of applying the time-window complexity for fault diagnosis is discussed. The mechanical signals of ball bearing with slight flaw and that of four kinds of impact-rubbing states of a typical rotor are then studied. The results show that time-window complexity can reflect the early fault of ball bearing, and differentiate the four kinds of rotor impact-rubbing states properly, which provides another effective way for fault diagnosis of mechanical systems.
\end{abstract}

Key Words: Complexity; Rolling bearing; Rotor rub; Early fault diagnosis

\section{Introduction}

At present, with the development of nonlinear science, complex systems and complexity science have become cross disciplinary research, there are many applications in the stochastic resonance feature description [1], biological signal processing [2] and power electronic system fault prediction [3]. Complexity theory and nonlinear research is closely related, before, people are accustomed to linarite the nonlinear system, but with the development of science, people gradually realized that this simplification is difficult to maintain the essence of the system. Complexity concept is proposed in this background, and nonlinear is the source of the complex behavior.

Mechanical signals directly reflect the dynamic behavior of mechanical system operation. The mechanical signal analysis, feature extraction to reflect the running state of the system is the key in fault diagnosis of mechanical equipment. The classical spectrum analysis method plays an important role in fault diagnosis, such as FFT analysis, holographic spectrum analysis, and wavelet analysis. However, these methods are given. The results are mechanical image of signal characteristics, depends largely on the experience, especially the mechanical system itself has a very strong nonlinear, non-stationary, makes the diagnosis more difficult. In order to realize the intelligent monitoring and diagnosis of machine operating, need some simple and the quantitative index to evaluate the mechanical equipment running reliable.

To solve this problem, many methods are raised at this moment; complexity analysis is one of a kind. Complexity provides a method for the analysis of time domain structure of mechanical signal, some qualitative, fuzzy concept, can be given as a quantitative description index. Complexity analysis can effectively monitoring system status and sensitively reflect the system fault, make up the deficiency of traditional monitoring methods. In the field of fault diagnosis for mechanical equipment, people have begun to use complexity method to analysis the mechanical signal, and achieved certain results [4].

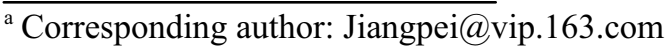


Due to non-uniform and non-stationary of signal's state space, will cause missing state information, in the extraction of signal characteristics, limits the application of complexity analysis [5]. The method of time window complexity can more effectively reflect the status of signal, to overcome the own limitations of complexity analysis, it will help to extract the characteristics of mechanical system at different fault distinguishing and early fault features. This paper focuses on the complexity and time window complexity concept, algorithm and its application in mechanical signal analysis. The time window complexity is used to study complexity of rolling bearing the early crack fault and rotor rub four running mechanical signals.

\section{Complexity concepts and algorithms}

In the study of complexity, there are many kinds of characterizations of the system complexity index, The Kolmogorov complexity, topological entropy, fractal dimension $\mathrm{D}$ and the formal language and automata are the famous represent [6,7]. And theoretical analysis and application results show that Kolmogorov complexity is very good at measuring the complexity.

Firstly, Kolmogorov defined the symbol sequence complexity concept in 1965 [8]. Kolmogorov complexity degree refers to the minimal number of bits required for a given " 0,1 " sequence with computer program, but this definition is difficult to establish a general algorithm. Until the Kasper Schuster [9], Lempel and Ziv [10] gave the algorithm of complexity. Lempel and Ziv complexity of a finite length sequence defined for with the length of the sequence increases the speed of new model.

The basic principle of the algorithm is as follows:

With the sequence $\left\{x_{i}\right\}=x_{1}, x_{2}, \mathrm{~L}, x_{N}$, this sequence is reconstructed:

$$
s_{i}= \begin{cases}1, & x_{i} \geq \bar{x} \\ 0, & x_{i}<\bar{x}\end{cases}
$$

Among of those, $\bar{x}=\left(x_{1}+x_{2}+\mathrm{L}+x_{N}\right) / N$

Based on the formula, the time series $\left\{x_{i}\right\}$ mapping sequence, $\left\{s_{i}\right\}=s_{1}, s_{2}, \mathrm{~L}, s_{N}$, which $s_{i}=0$ or 1 .

Kolmogorov think that the complexity of $\left\{s_{i}\right\}$ reflect the complexity of $\left\{x_{i}\right\}$. For the formation of the "0,1" sequence, Lempel and Ziv attributed the two kinds of operations: the first is "copy", namely a prefix of sequence produce a longer prefix; the second is "add", namely add symbols to the prefix had been generated, any length $\mathrm{n}$ sequences of symbols can be produced by the second operation from an empty string, but only the shortest description is the complexity of sequences.

First of all, from the empty string begin to generate. A prefix have been generated shilling, $S=\left(s_{1}, \mathrm{~L} s_{r}\right), r<N$. Assumed, $Q=s_{r+1}$ formed SQ string, $S Q \pi$ is the string after deleting the last character from the string $S Q$. If $Q$ can be copied from a sub string from $S Q \pi$, put the character to be added later, known as "copy", if not belonging to, is said to "add" and "add" with a "." before and after the separation. If $Q=s_{r+1}$ can be obtained from a sub string $S Q \pi$ by "copy", then continue to investigate whether $Q=S_{r+1} S_{r+2}$ obtained from a sub string $S Q \pi$ by "copy". If not, belong to "add", $S=\left(s_{1}, \mathrm{~L} s_{r} s_{r+1} S_{r+2} \bullet\right)$, so that in the back of $s_{1} \mathrm{~L} s_{r} S_{r+1} s_{r+2}$ with ".". Next, all characters in front of"." look as S. Repeat the above steps until generating the entire sequence.

For example: generate " 0,1 " sequence " $0110 "$.

(1) To generate a character by adding from the empty string denoted as $\rightarrow 0 \bullet$.

(2) $S=0, Q=1, S Q=01, S Q \pi=0, \quad Q$ not belongs to $S Q \pi$, noted as $\rightarrow 0 \bullet 1 \bullet$.

(3) $S=01, Q=1, S Q=011, S Q \pi=01, \quad Q$ belongs to $S Q \pi$, noted as $\rightarrow 0 \bullet 1 \bullet 1$.

(4) $S=01, Q=10, S Q=0110, S Q \pi=001, Q$ not belongs to $S Q \pi$, 记为 $\rightarrow 0 \bullet 1 \bullet 10 \bullet$.

In this way, we get a string divided into segments by ".", the number of segments is defined as the complexity of $\mathrm{C}$, so the complexity of sequence " 0010 " note as $C(4)=3$.

According to the Lempel and Ziv, almost all 0,1 random sequences, their complexity will tend to a value $\mathrm{b}(\mathrm{N})$ :

$$
b(N)=\lim _{N \rightarrow \infty} C(N) \equiv N / \log _{2} N
$$

Next, normalization, so that it becomes the relative complexity of $C_{r}(N)$. 


$$
C_{r}(N)=C(N) / b(N)
$$

We can find that relatively complexity $C_{r}(n)$ reflects similarity degree between a time series and random sequence, the $\operatorname{Cr}(n)$ of completely random sequence will tends to 1 , and periodic sequence will be $0, \operatorname{Cr}(N) \in[0,1]$ when series between the two cases. Thus, signal complexity is related with signal ordered and random behavior, relatively complexity $C_{r}(n)$ can be the characteristic parameters to represents the state of the system.

Complexity of system is very sensitive, when the machinery and equipment have early fault, complexity analysis of changes can reflect changes in the state of the system, and complexity is especially suitable for computer processing. In this paper, complexity analysis is used to detect the early crack of bearing inner ring and early touch rub of rotor.

\section{Early crack fault detection of rolling bearing inner} ring

Rolling pair local defects is the most typical failure mode of rolling bearing; especially it is most difficult to diagnose the inner ring rolling defects. This is because that the impact has a transmission link caused by raceway defects, the fault feature signal is transmitted to the base surface has been greatly weakened, flooded in background noise. It is not useful to diagnose the faults of rolling bearing by the characteristic frequency.

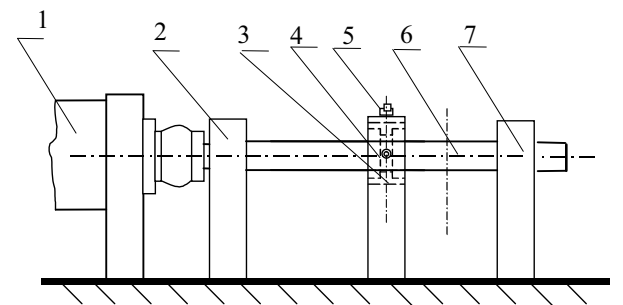

1- motor 2- sliding bearing 3- rolling bearing 4-

horizontal direction (x) displacement sensor 5-vertical direction (y) displacement sensor 6- shaft 7- support

Figure 1. Experimental rotor-bearing rig

As shown in figure 1, the normal state and early crack failure state are all tested in the rotor bearing test.

In speed $3500 \mathrm{r} / \mathrm{min}$, the sampling frequency is $2.5 \mathrm{kHz}$; we get 10000 sampling points in horizontal and vertical direction. Then signal is processed by low-pass filtering, filter cutoff frequency is $1 \mathrm{kHz}$. Waveform and power spectrum in the state of fault and the inner crack at early stage are given respecti vely in the Figure 2.
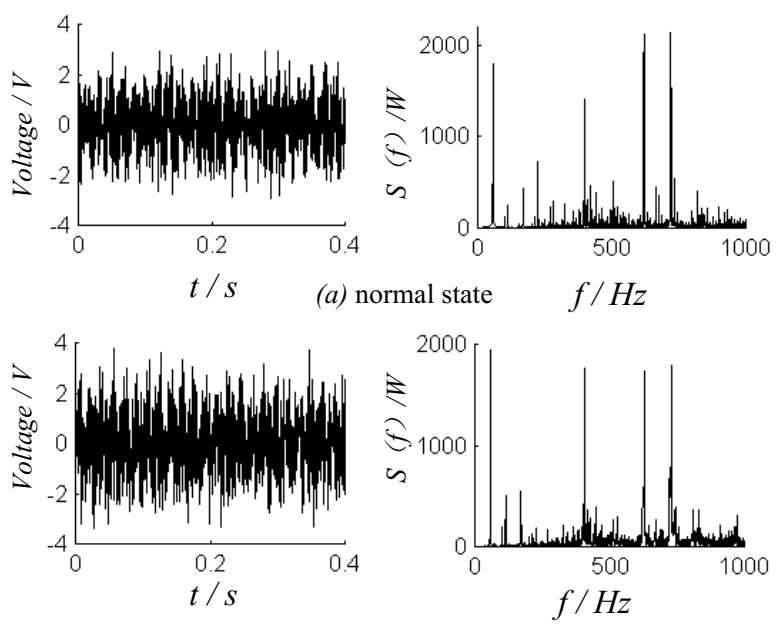

(b) Early crack of inner ringnormal state

Figure 2. Waveform and spectrum of ball bearing vibration signals

From the above, we could not tell the differenc e between rolling bearing inner ring raceway early c rack fault and no fault condition in the time domain and frequency domain. Following the complexity an alysis is used to analyzed the 5 groups of sampled data, the length of each set of data for 2000 points, complexity are calculated in table 1.

Table 1. Relative complexity of ball bearing vibration signals

\begin{tabular}{llllll}
\hline & & \multicolumn{4}{c}{ Relative complexity $C_{r}$} \\
\cline { 2 - 6 } $\begin{array}{l}\text { Bearing } \\
\text { condition }\end{array}$ & First & Second & Third & Fourth & Fifth \\
& groups & groups & groups & groups & groups \\
\hline failure-free & 0.740 & 0.738 & 0.762 & 0.733 & 0.742 \\
& 6 & 5 & 1 & 7 & 8 \\
Early crack & 0.857 & 0.853 & 0.857 & 0.860 & 0.858 \\
& 4 & 8 & 7 & 6 & 1 \\
\hline
\end{tabular}

The complexity of fault is more complex. Thus, it could be effective to extract the fault characteristic of rolling bearing inner ring raceway early crack fault detection by complexity analysis.

\section{Early rub impact fault detection}

Next, rotor rubs experiment is taken on the on test bench. Rotor frequent 3500r/min, axis horizontal and vertical displacement is measured by the measurement system using KEYENCE-LC2401 laser displacement sensor. Signal is also processed by low-pass filtering, filter cutoff frequency is $800 \mathrm{~Hz}$, and sampling frequency $5 \mathrm{KHz}$ sampling number is 10000 .

In the rub friction experiment, the signal is strong non-stationary between the rotor and the stator occurred 
early rub impact (the beginning touch). And, complex degree analysis can reflect the early rub impact fault of rotor. Waveform and the axis orbit of horizontal direction of signal are given without rubbing and early rub impact in Figure 3 (a) and (b).
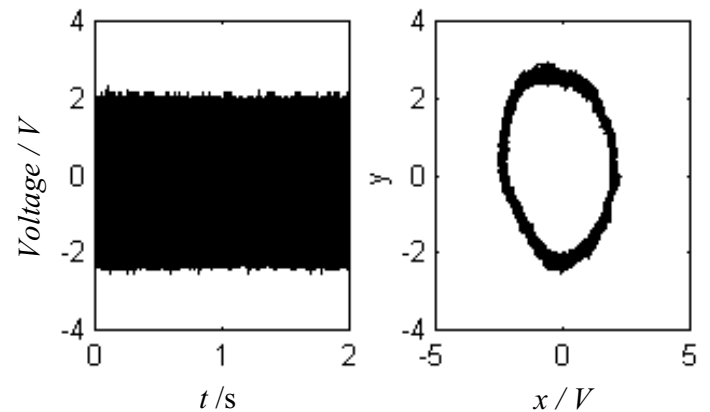

(a) No impact friction

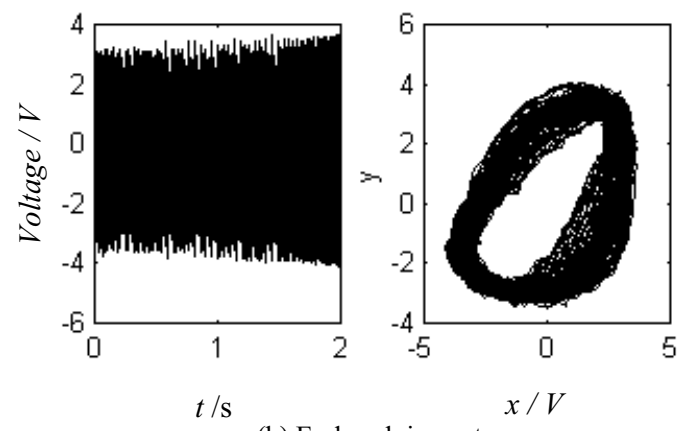

(b) Early rub impact

Figure 3. Waveform and axes track of rotor rub signals

From the figure, the shaft center trajectory becomes complicated and can qualitatively reflect the early rub impact conditions, but without a clear quantitative indicators. Following the relative complexity of multiple sets of data obtained by sampling analysis, each data length of 2000. Results are shown in Table 2.

From table 2, it can be seen that the signal of no rotor rub impact and early rub friction relative complexity is significantly different. The complexity of no rotor rub is smaller than early rub friction signal. So that, according to the relatively complex of signal, we can identify early detection of rub fault.

Table 2. Relative complexity of rotor rubs signals

\begin{tabular}{clllll}
\hline & \multicolumn{5}{c}{ Relative complexity $C_{r}$} \\
\cline { 2 - 6 } $\begin{array}{c}\text { Rub impact } \\
\text { condition }\end{array}$ & First & Second & Third & Fourth & Fifth \\
& groups & groups & groups & groups & groups \\
\hline failure-free & 0.102 & 0.101 & 0.098 & 0.106 & 0.104 \\
& 0 & 3 & 7 & 8 & 3 \\
Early crack & 0.132 & 0.134 & 0.131 & 0.139 & 0.136 \\
& 5 & 8 & 1 & 8 & 0 \\
\hline
\end{tabular}

5 conclusions

This paper introduces the concept and calculation method of time window complexity, and the bearing inner ring early crack fault signal and rotor rub fault signal of rolling are analyzed in this paper. The analysis results show that there is no obvious difference in the time domain and frequency domain between the mechanical signal of rolling bearing inner ring occurred early crack and free fault state, but the former time window complexity index is significantly higher than the latter; Another window complexity can overcome alone with complex index as characteristic parameters, can describe the strong characterizations of the running state of machine, which provide a method to extract fault features from mechanical signals, further provide a effective method to fault diagnosis.

\section{References}

1. P. Shi, X. Ding, and D. Han. Study on multi-frequency weak signal detection method based on stochastic resonance tuning by multi-scale noise, Measurement, 2014,47(1): 540-546

2. Moses O. Sokunbi, George G. Cameron, Trevor S. Ahearn, Alison D. Murray, Roger T. Staff. Fuzzy approximate entropy analysis of resting state fMRI signal complexity across the adult life span, Medical Engineering \& Physics, 2015,37(11): 1082-1090

3. Liu Qing, Wang Zengping, Zheng Zhenhua, Chang Yanyan. On-unit Transient Protection Based on Signal Complexity for Series Compensated Transmission Lines, Proceedings of the CSEEN, 2010,30(13): $81-88$

4. Xiaoli Li, Gaoxiang Ouyang, Zhenhu Liang. Complexity measure of motor current signals for tool flute breakage detection in end milling, International Journal of Machine Tools and Manufacture, 2008,48(3): 371-379

5. Long Fei, Zhang Daoxin, Fan Ling, Wu Xiaopei. Analysis of sleep staging by time-window complexity sequence of EEG.. Journal of Anhui University Natural Science Edition, 2002,26(1): 56-61

6. Grassberger. Toward a quantitative theory of self-generated complexity. Int. J.Theroy.Phys.,1986,25: 907-936.

7. B.L.Hao. Symbolic dynamics and characterization of complexity. Physica D, 1991,51,161-176.

8. R. Wackerbauer, et al A comparative classification of complexity measures. Chaos, Solitons \& Fractals,1994, 
4(1): 133-173.

9. F.Kasper, H.G.Schuster. Easily calculable measure for the complexity of spatiotemporal patterns. Physical review A, 1987,36(2): 842-848.

10. Abraham Lempel, Jacob Ziv. On the complexity of finite sequences. IEEE Transactions on information theory,1976, 22(1): 75-81. 